\title{
Introduction: \\ Exploring Nordic Game Research
}

\author{
Raine Koskimaa \\ University of Jyväskylä \\ raine.koskimaa@jyu.fi
}

\section{Frans Mäyrä}

University of Tampere

frans.mayra@uta.fi

\section{Jaakko Suominen}

University of Turku

jaakko.suominen@utu.fi

Nordic DiGRA 2012 Conference was held at the University of Tampere on June 6-8, 2012. In this Special Issue of the Transactions of DiGRA journal, we present a selection of the best papers of that conference.

The study of games, players and related phenomena has increased both in its academic and geographical range during the last decade. The Nordic DiGRA 2012 conference aimed to bring together Nordic and non-Nordic games researchers to discuss the cultural and social context of games and play from multiple perspectives. 2012 marked the tenth anniversary of the Tampere Computer Games and Digital Cultures (CGDC 2002) conference, which also provided an opportunity to reflect on the last decade of development in Nordic game studies, as well as its wider international scope.

Out of the 47 submissions, there were 24 papers selected to the conference after a double blind review of full papers. The article by Simo 
Järvelä \& al., "A Practical Guide to Using Digital Games as an Experiment Stimulus," received the Nordic DiGRA 2012 Best Paper Award. In addition, there were two panels, one round table discussion, and a special track (not subjected to the review process) on gambling studies. The majority of papers came from the Nordic countries, but altogether 15 nationalities were present in the list of speakers. Nick Montfort (USA), Espen Aarseth (Norway/Denmark), Minna Rückenstein (Finland), and Kristine Jørgensen (Norway) delivered the keynotes by invitation. Out of the track themes, most popular were "design of games," "social player studies," "games as media and communication," and "critical approaches to game studies."

The collection of articles presented here is based on the conference review process; they represent the strongest contributions of the conference. In addition, there has been a rigorous and thorough review process during journal publication. Since the conference presentations, articles have undergone substantial revisions. We would like to express our gratitude for the conference reviewers, ToDiGRA journal reviewers, and the ToDIGRA editors, for their invaluable efforts in reviewing the papers. Our special thanks to the authors who have put much effort in writing and rewriting their articles to meet the ToDiGRA format and standards.

The first article, by Jaakko Stenros, reviews the history of the concept of the magic circle, its criticism and other metaphors that have been used to capture the zone of play such as world, frame, bubble, net or attitude. The various conceptions of social, mental and cultural borders are reviewed and criticisms toward the concept of magic circle are addressed. As a result, a model is presented in which the psychological bubble of playfulness, the social contract of the magic circle and the cultural game forms are separated.

In their article "Player Types: A Meta-synthesis" Hamari and Tu- 
unanen investigate the ways in which players have been typified in research literature. Their aim is to distinguish typologies relevant to researchers and for designing and marketing games. By synthesizing the results of various studies and comparing them, they draw implications for further studies. The previous works on player typologies are further analyzed using a concept-centric approach and synthesized according to common and repeating factors in the previous studies. As a result the player types in previous literature are synthesized into seven primary dimensions: intensity, achievement, exploration, sociability, domination, immersion and in-game demographics.

Linderoth, Björk and Olsson, in "Should I Stay or Should I Go? A Study of Pick Up Groups in Left 4 Dead 2," report an autoethnographic study of pick up groups (groups formed organically by players at the time of play) in the game Left 4 Dead 2. The study focuses on pick up groups as social arenas and the role of game design in structuring interaction. They analyze issues such as inclusion and exclusion, social position and the relation between the game context and the players' "wider worlds." The study tentatively suggests that positive perceptions of other players' out-of-game identity can save a game from falling apart, yet negative perceptions of other players' values and out-of-game identities pose no threat to the game activity.

"Player-reported Impediments to Game-based Learning," by Harviainen, Lainema and Saarinen, addresses the question of how games function as learning tools from the perspective of player-stated problems. It is based on interviews and essays, collected from university students who reported problems dealing with unrealistic levels of trust and competitive play. These players searched for the logic in the game as an artifact instead of considering decision alternatives applicable to real-world situations. The main source of difficulty appeared to be that for many participants, games are framed as an activity that is to be done competitively. Along with reporting the impediments, the article 
discusses potential solutions.

Digital games have proved useful as a stimulus in research settings. "A Practical Guide to Using Digital Games as an Experiment Stimulus," by Järvelä, Ekman, Kivikangas and Ravaja, examines the advantages and challenges of using games in experimental research with particular focus on strict stimulus control in matching and regulating task type, data segmentation and event coding; compatibility between participants; and planning and conducting data collection. They provide a breakdown of the steps necessary for using a digital game in experimental studies and offer a checklist for researchers to account for the reliability and validity of the experiments. The article also provides a case study illustrating how their considerations apply in practice. Digital gameplay is now firmly embedded in everyday practices in many Scandinavian homes. Enevold's article "Digital Materialities and Family Practices: the Gendered, Practical, Aesthetical and Technological Domestication of Play" deals with the constitution of gaming practices in families, focusing especially on the material objects essential to play and their role in everyday life. Enevold uses ethnographic methods and anthropological practice theory to attend to the domestic spaces of leisure and play, the home environment, in which a large part of today's practices of playing digital games take place. She focuses on the staging of material artifacts of gaming and demonstrates how everyday practices order space-time and artifacts while domesticating play and conditioning performances of family, gender and gaming. The article also provides a history of the domestication of play.

The Nordic DiGRA 2012 Conference also served as the closing event for "The Creation of Game Cultures - A Case of Finland," a major research project funded by the Academy of Finland and carried out by a consortium of partners from the Universities of Tampere, Turku and Jyväskylä. The project resulted in an improved understanding of playing and gaming habits in Finland. Issues such as how much traditional 
and digital games are played in Finland, who are the primary players of various game types, and what are the main types of playing activity have been investigated in the Player Barometer survey series initiated in 2009 as part of the project. According to the latest Player Barometer almost all Finns (98\%) play at least some form of game (traditional or digital) sometimes, and most of them (89\%) could be classified as "active players," meaning that they reported playing at least one form of game at least once a month. When digital games are more closely scrutinized, active digital gamers have already grown to be majority in Finland (54\%). In terms of age, the average digital gamer continues to approach the average of entire population; in our 2011 findings, the average age of a digital game player was 37 years (for more details, see Karvinen \& Mäyrä 2011).

The project also resulted in the creation of even more multidisciplinary dialogue in game studies, especially bridging the gaps between gambling and entertainment play as well as between digital and non-digital game play. This is important especially in light of how popular gambling (or 'money gaming') is in Finland: according to our survey active gamblers form a majority (55\%) of the Finns.

In the Nordic countries, the role of games is not fixed but rather changing dynamically. In Finland, as we closely examined the evidence of our three-year survey data, we noticed that the oldest age group (70-75 years) had increased their game playing in statistically significant manner when all forms of games were considered. Active players constituted $75 \%$ of this age group in the 2009 sample, but in 2011 this figure had risen to $91 \%$. Clearly either the older generation is becoming more interested in games, or they have started to report their game playing more openly - both explanations are important. We also found that digital game playing had increased from 2009 to 2011. In 2009 there were $51 \%$ of the sample qualified as active digital game players, whereas in 2011, active gamers increased to $56 \%$ of the 
sampled population. These findings have also been incorporated in a recent international comparison between gaming habits in various countries (Quandt, Chen, Mäyrä \& van Looy 2013).

Topics such as the merging of the social functionalities of gambling and entertainment play, mapping out of the Nordic LARP scene, "social games," games as part of history culture, the relation of digital play to digital sports, archival and museum presentation of games and related artifacts, game realism, game narratives, and games as signifying structures were addressed in the project. This list, together with the wide variety of topics included in the Nordic DiGRA 2012 Conference (conference papers are available in the DiGRA Digital Library) give a good sense of the richness and activity in game studies in the Nordic region. We offer this selection of articles as a window to that lively scene.

\section{BIBLIOGRAPHY}

Karvinen, J. and F. Mäyrä. Pelaajabarometri 2011: Pelaamisen muutos. [Player Barometer 2011: changing play; with English abstract]. TRIM Research Reports 6. University of Tampere: Tampere, 2011. Available at: http://urn.fi/urn:isbn:978-951-44-8567-1 Quandt, T., Chen,V., Mäyrä, F. \& van Looy, J. (Multiplayer) Gaming Around the Globe? A Comparison of Gamer Surveys in Four Countries. In Quandt, T. and S. Kröger. (eds.)(2013) Multiplayer: The Social Aspects of Digital Gaming. Routledge: London, $\mathrm{p}$ 23-46. 